\title{
Fragmented Parathyroid Hormone Measurement
}

National Cancer Institute

\section{Source}

National Cancer Institute. Fragmented Parathyroid Hormone Measurement. NCI

Thesaurus. Code C74784.

The determination of the amount of fragmented parathyroid hormone present in a sample. 